\title{
Absolute 10-year risk of dementia by age, sex and $A P O E$ genotype: a population-based cohort study
}

\author{
Katrine L. Rasmussen MD PhD, Anne Tybjærg-Hansen MD DMSc, Børge G. Nordestgaard MD DMSc, \\ Ruth Frikke-Schmidt MD DMSc
}

Cite as: CMAJ 2018 September 4;190:E1033-41. doi: 10.1503/cmaj.180066

\section{ABSTRACT \\ BACKGROUND: Dementia is a major cause of disability, and risk-factor reduction may have the potential to delay or prevent the disease. Our aim was to determine the absolute 10-year risk of dementia, by age, sex and apo- lipoprotein $\mathrm{E}(A P O E)$ genotype.}

METHODS: We obtained data from the Copenhagen General Population Study (from 2003 to 2014) and the Copenhagen City Heart Study (from 1991 to 1994 and 2001 to 2003). Participants underwent a questionnaire, physical examination and blood sampling at baseline. Diagnoses of dementia and cerebrovas- cular disease were obtained from the Danish National Patient Registry up to Nov. 10, 2014.

RESULTS: Among 104537 individuals, the absolute 10-year risk of Alzheimer disease in 3017 women and men who were carriers of the APOE $\varepsilon 44$ genotype was, respectively, $7 \%$ and $6 \%$ at age $60-$ 69 years, $16 \%$ and $12 \%$ at age $70-$ 79 years, and $24 \%$ and $19 \%$ at age 80 years and older. Corresponding values for all dementia were $10 \%$ and $8 \%, 22 \%$ and $19 \%$, and $38 \%$ and $33 \%$, respectively. Adjusted hazard ratios (HRs) for all dementia increased by genotype, from genotype $\varepsilon 22$ to $\varepsilon 32$ to $\varepsilon 33$ to $\varepsilon 42$ to $\varepsilon 43$ to $\varepsilon 44$ ( $p$ for trend $<0.001$ ). Compared with $\varepsilon 33$ carriers, $\varepsilon 44$ carriers were more likely to develop Alzheimer disease (adjusted HR 8.74, 95\% confidence interval [Cl] 7.08-10.79), vascular dementia (adjusted HR 2.87, 95\% Cl 1.54-5.33), unspecified dementia (adjusted HR 4.68, $95 \% \mathrm{Cl} 3.74-5.85)$ and all dementia (adjusted HR 5.77, 95\% Cl 4.89-6.81).

INTERPRETATION: Age, sex and $A P O E$ genotype robustly identify high-risk groups for Alzheimer disease and all dementia. These groups can potentially be targeted for preventive interventions.

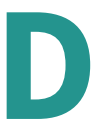

ementia is a major cause of disability in later life, and the global prevalence is increasing, especially in lowand middle-income countries. ${ }^{1,2}$ Reduction of risk factors for dementia may have the potential to delay or prevent one-third of dementia cases. ${ }^{3}$ The estimation of absolute risk specific to age, sex and apolipoprotein $\mathrm{E}(A P O E)$ genotype to identify high-risk individuals for targeted prevention is needed. Cerebrovascular disease is similarly a major cause of both morbidity and mortality, ${ }^{1,4}$ and is closely associated with development of dementia.

The apolipoprotein $\mathrm{E}$ (apoE) protein is pivotal for peripheral and cerebral cholesterol metabolism as well as for cerebral clearance of amyloid- $\beta$ in Alzheimer disease..$^{5-7}$ The $\varepsilon 4$ allele of the $A P O E$ polymorphism is by far the strongest genetic risk factor implicated in common late-onset Alzheimer disease, ${ }^{8}$ an association first recognized in $1993,{ }^{9}$ and since validated globally. ${ }^{10}$
Three human apoE isoforms, apoE2, apoE3 and apoE4, corresponding to 3 common alleles, $\varepsilon 2$, $\varepsilon 3$ and $\varepsilon 4$, defined by 2 single

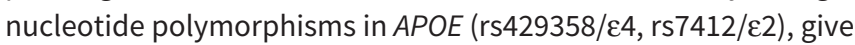
rise to 6 common $A P O E$ genotypes, $\varepsilon 22, \varepsilon 32, \varepsilon 33, \varepsilon 42, \varepsilon 43$ and $\varepsilon 44$. Whereas compelling evidence suggests that the APOE polymorphism is involved in risk of Alzheimer disease, the role for subtypes of dementia as well as for cerebrovascular disease - a major risk factor for dementia - is less clear despite repeated previous studies..$^{5,911-19}$ In particular, the estimation of 10-year absolute risk for dementia specific to age, sex and APOE genotype are lacking, information that is essential for providing targeted prevention against dementia in high-risk individuals.

We sought to determine the 10-year absolute risk of Alzheimer disease, vascular dementia and all dementia, stratified by age, sex and $A P O E$ genotype, as well as associations between $A P O E$ genotype and cerebrovascular disease. 


\section{Methods}

\section{Participants}

We included individuals from 2 similar prospective studies of the Danish general population: the Copenhagen General Population Study and the Copenhagen City Heart Study. ${ }^{20-23}$ The Copenhagen General Population Study was initiated in 2003 with the first enrolment period from 2003 to 2015, and with follow-up examinations ongoing. The Copenhagen City Heart Study was initiated in 19761978 with follow-up examinations in 1981-1983, 1991-1994 and 2001-2003. Data collection included a questionnaire, a physical examination, and blood sampling for biochemical and DNA analyses. Individuals were randomly selected from the national Danish Civil Registration System to reflect the adult population aged 20 years and older. There was no overlap of individuals between studies. All participants were white and of Danish descent. The studies combined included a total of 104537 individuals, of whom 2160 developed dementia and 7520 developed cerebrovascular disease. Descriptions of the cohorts and definitions of clinical variables are detailed in Appendix 1, available at www.cmaj.ca/ lookup/suppl/doi:10.1503/cmaj.180066/-/DC1.

\section{Dementia and cerebrovascular endpoints}

Information on diagnoses was collected from the Danish National Patient Registry, with data on all patient contacts from all clinical hospital departments in Denmark since 1977, and from the National Danish Registry of Causes of Death, with data on causes of all deaths in Denmark, as reported by hospitals and general practitioners since 1977 .

We defined Alzheimer disease as the International Classification of Diseases (ICD) 8th and 10th revision codes ICD-8 290.10 and ICD-10 F00 and G30, vascular dementia as ICD-10 F01, and unspecified dementia as ICD-8 290.18 and ICD-10 F03. All dementia included Alzheimer disease, vascular dementia and unspecified dementia. Information on cerebrovascular disease (ICD-8 431-438 and ICD-10 160-169, G45) is described in Appendix 1.

For Cox regression models for APOE genotypes, follow-up began at birth or at the start of the registries (Jan. 1, 1977), whichever came last. For all other analyses, follow-up began at the time of blood sampling (2003 and onwards for the Copenhagen General Population Study, and 1991-1994 or 2001-2003 for the Copenhagen City Heart Study). Follow-up ended at occurrence of event, death, emigration or on Nov. 10, 2014 (last update of the registries), whichever came first. Median follow-up from start of the registries was 38 (range 0-38) years for both all dementia and cerebrovascular disease, with no individuals lost to follow-up.

\section{Biochemical and genetic analyses}

Biochemical and genetic analyses are described in Appendix 1. Based on prior studies and understanding of biological mechanisms, we hypothesized that associations between $A P O E$ genotype and risks of Alzheimer disease and all dementia would increase from $\varepsilon 22$ to $\varepsilon 32$ to $\varepsilon 33$ to $\varepsilon 42$ to $\varepsilon 43$ to $\varepsilon 44$. Because the ع42 genotype represents 2 hyperlipidemic alleles, ${ }^{7}$ we hypothesized that risk for vascular endpoints - vascular dementia and cerebrovascular disease - would increase from $\varepsilon 22$ to $\varepsilon 32$ to $\varepsilon 33$ to $\varepsilon 43$ to $\varepsilon 42$ to $\varepsilon 44$. Finally, because the apoE2 isoform is defective in binding to the low-density lipoprotein (LDL) receptor, genotypes harbouring the $\varepsilon 2$ allele have the highest plasma apoE levels. Hence, trends in lipoprotein and apolipoproteins are hypothesized to be associated with a decreasing trend in plasma apoE levels from $\varepsilon 22$ to $\varepsilon 32$ to $\varepsilon 42$ to $\varepsilon 33$ to $\varepsilon 43$ to $\varepsilon 44$. Consequently, the results are organized according to the hypothesized hierarchies of associations.

\section{Statistical analysis}

Absolute 10-year risk of Alzheimer disease and all dementia as a function of $A P O E$ genotype and stratified by age (in 3 age groups) and by sex was estimated by competing risk models. ${ }^{24}$ Data were presented as estimated incidence rates (number of events per 10 years) in percent. Cumulative incidence curves and respective trends across genotypes were generated from competing risk regression models, according to the method of Fine and Gray. ${ }^{24}$ Cumulative incidence was further evaluated by Kaplan-Meier curves and log-rank trend tests. To test whether $A P O E$ genotypes were associated with increased risk of dementia and cerebrovascular disease, we used Cox regression models adjusted for known biologically relevant risk factors and markers of lifestyle regardless of the sizes of their contributions. Additional details regarding the statistical analysis are described in Appendix 1.

\section{Ethics approval}

The Copenhagen General Population Study and the Copenhagen City Heart Study were approved by the Committee of Biomedical Research Ethics for Copenhagen, Denmark and relevant institutional review boards, and were conducted according to the Declaration of Helsinki, with written informed consent from participants. Separate ethics approval was not required for use of these data in this study.

\section{Results}

Baseline characteristics of the 104537 individuals by APOE genotype are shown in Table 1 . Levels of covariates were similar across APOE genotypes, except for lipid-lowering treatment, which varied significantly from $7 \%$ to $15 \%(p<0.001)$. This finding was expected, as APOE genotype affects lipid levels.

\section{0-year absolute risk for dementia}

For Alzheimer disease, 10-year absolute risk increased by genotype, from $\varepsilon 32$ to $\varepsilon 33$ to $\varepsilon 42$ to $\varepsilon 43$ to $\varepsilon 44$, whereas $\varepsilon 22$ had a slightly higher 10-year absolute risk than $\varepsilon 32$ and $\varepsilon 33$ in both sexes across all age groups (Figure 1). Ten-year absolute risk of all dementia increased from $\varepsilon 22$ to $\varepsilon 32$ to $\varepsilon 33$ to $\varepsilon 42$ to $\varepsilon 43$ to $\varepsilon 44$ in both sexes across all age groups. The absolute 10 -year risks for ¿44 genotype for Alzheimer disease for age groups 60-69 years, $70-79$ years, and 80 years or older were $7 \%, 16 \%$ and $24 \%$ for women, and $6 \%, 12 \%$ and $19 \%$ for men, respectively. Corresponding percentages for all dementia were $10 \%, 22 \%$ and $38 \%$ for women, and $8 \%, 19 \%$ and $33 \%$ for men. Percentages for vascular dementia were substantially lower, all below 3\% (Figure 1). 


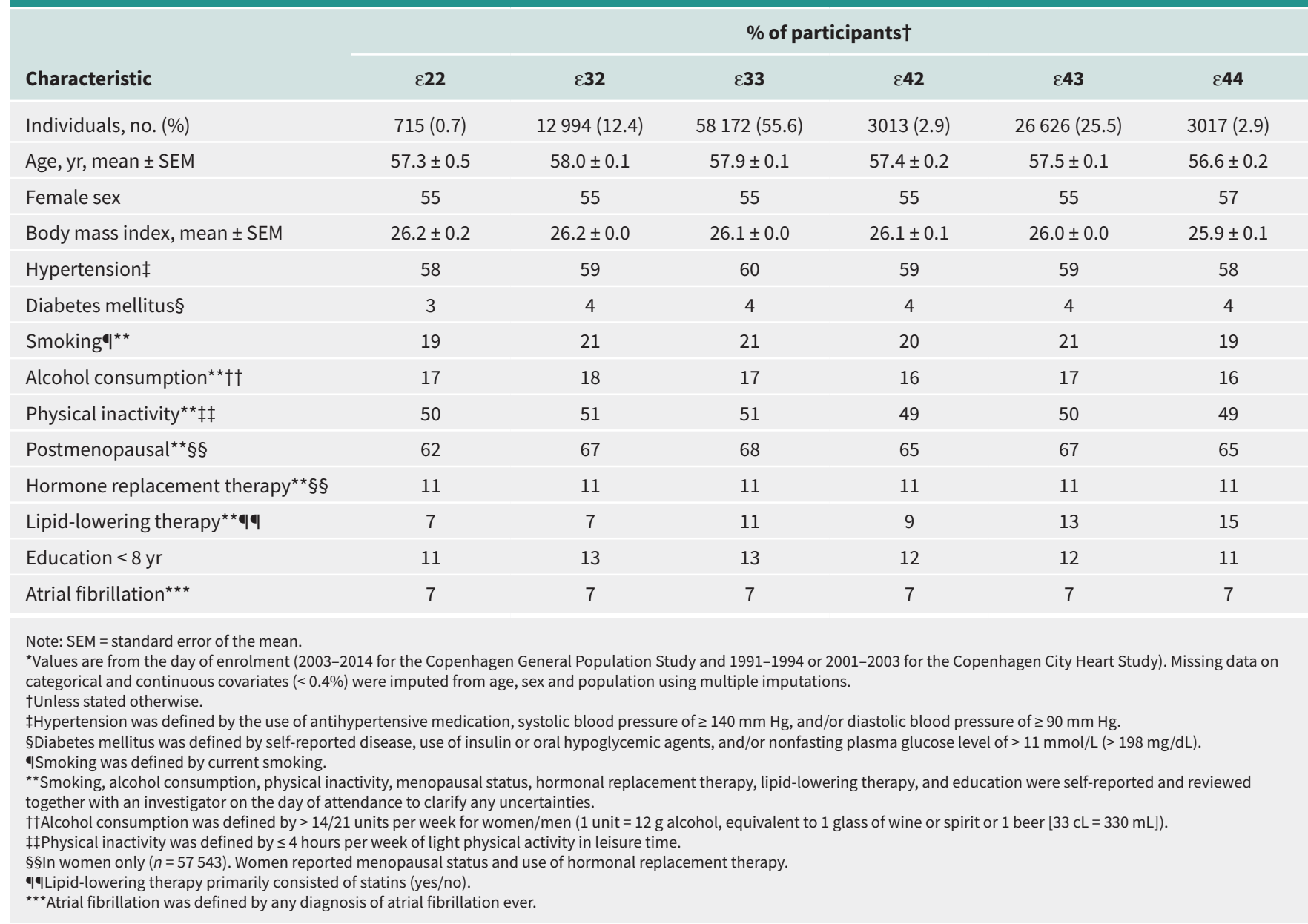

\section{$A P O E$ genotype and risk of dementia}

Cumulative incidence of Alzheimer disease, unspecified dementia and all dementia increased with increasing number of $\varepsilon 4$ alleles from $\varepsilon 42$ to $\varepsilon 43$ to $\varepsilon 44$ ( $p$ for trend $<0.001$ for all). In contrast, cumulative incidence of vascular dementia was highest in $\varepsilon 44$ and $\varepsilon 42$ genotypes, and lowest for $\varepsilon 22$ genotype and hardly increased with age ( $p$ for trend $=0.04$ ) (Figure 2). Multivariable-adjusted hazard ratios (HRs) increased from $\varepsilon 32$ to $\varepsilon 33$ to $\varepsilon 42$ to $\varepsilon 43$ to $\varepsilon 44$ for Alzheimer disease, from $\varepsilon 33 / \varepsilon 32$ to $\varepsilon 43$ to $\varepsilon 42$ to $\varepsilon 44$ for vascular dementia, and from $\varepsilon 22$ to $\varepsilon 32$ to $\varepsilon 33$ to $\varepsilon 42$ to $\varepsilon 43$ to $\varepsilon 44$ for unspecified dementia and all dementia ( $p$ for trends $<0.001$ for all) (Figure 3). Estimates were similar for age- and sexadjusted analyses (Appendix 1, Supplemental Table 1) after additional adjustments for lipids and lipoproteins, when entry at time of blood sampling was used, and when competing risk models were used (data not shown). Interaction terms between $A P O E$ genotype and sex reached significance for Alzheimer disease $(p=0.03)$ and all dementia $(p=0.05)$ (Appendix 1, Supplemental Table 2). Estimates for vascular dementia remained similar after exclusion of 45 individuals with both Alzheimer disease and vascular dementia (Appendix 1, Supplemental Figure 1).
Other covariates independently associated with an increased risk of all dementia included smoking (adjusted HR 1.68, 95\% confidence interval $[\mathrm{Cl}] 1.52-1.85$ ), less than 8 years of education (adjusted HR 1.34, 95\% Cl 1.23-1.47), diabetes mellitus (adjusted HR $1.30,95 \% \mathrm{Cl} 1.09-1.55)$ and physical inactivity (adjusted HR $1.16,95 \% \mathrm{Cl} 1.06-1.27$ ). In contrast, male sex (adjusted HR 0.62, $95 \% \mathrm{Cl} 0.40-0.96$ ), lipid-lowering therapy (adjusted $\mathrm{HR} 0.81,95 \% \mathrm{Cl}$ $0.71-0.93$ ) and hypertension (adjusted HR $0.82,95 \% \mathrm{Cl} 0.74-0.91$ ) were associated with a decreased risk of all dementia (Table 2).

\section{APOE genotype, cerebrovascular disease, and lipids, lipoproteins and apolipoproteins}

Multivariable-adjusted HRs were $1.22(95 \% \mathrm{Cl} 1.01-1.48)$ and 1.31 (95\% $\mathrm{Cl} 1.08-1.58)$ for $\varepsilon 42$ and $\varepsilon 44$ versus $\varepsilon 33$ for ischemic stroke (Appendix 1, Supplemental Figure 2). Similarly, HRs were 1.19 (95\% Cl 1.03-1.37) and 1.29 (95\% Cl 1.12-1.48) for ischemic cerebrovascular disease, $1.15(95 \% \mathrm{Cl} 0.78-1.71)$ and $1.81(95 \% \mathrm{Cl}$ 1.29-2.53) for hemorrhagic stroke, and 1.17 (95\% Cl 1.03-1.34) and $1.30(95 \% \mathrm{Cl} 1.15-1.48)$ for all cerebrovascular disease for $\varepsilon 42$ and $\varepsilon 44$ versus $\varepsilon 33$ (Appendix 1, Supplemental Figure 2). Estimates were similar after adjustment for lipids and lipoproteins, when entry at time of blood sampling was used, and when competing risk models were used (data not shown). 

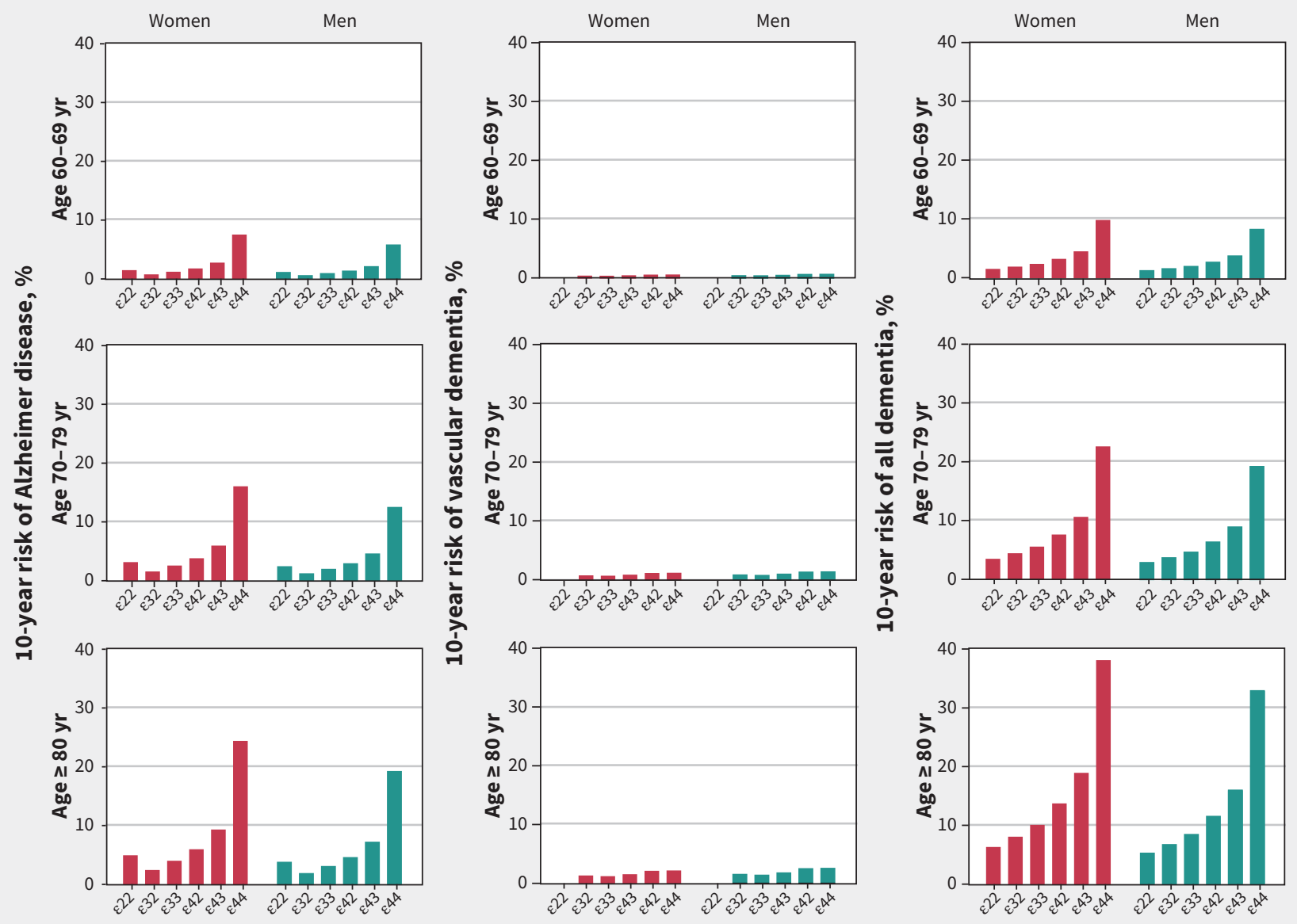

Figure 1: Absolute 10-year risk of Alzheimer disease, vascular dementia and all dementia for APOE genotypes stratified by sex and age groups. Competing risk was accounted for by the method of Fine and Gray. ${ }^{24}$

Risk of all dementia after a diagnosis of cerebrovascular disease increased from $\varepsilon 32$ to $\varepsilon 33$ to $\varepsilon 43$ to $\varepsilon 42$ to $\varepsilon 44$ ( $p$ for trend = 0.002) (Appendix 1, Supplemental Figure 3). A similar pattern was observed for a multivariable-adjusted competing risk model (Appendix 1, Supplemental Figure 3).

For the 6 common $A P O E$ genotypes, total cholesterol, LDL cholesterol and apolipoprotein B increased from $\varepsilon 22$ to $\varepsilon 32$ to $\varepsilon 42$ to $\varepsilon 33$ to $\varepsilon 43$ to $\varepsilon 44$ ( $p$ for trend $<0.001$ for all), whereas highdensity lipoprotein cholesterol and apolipoprotein Al levels decreased from $\varepsilon 22$ to $\varepsilon 32$ to $\varepsilon 42$ to $\varepsilon 33$ to $\varepsilon 43$ to $\varepsilon 44$ ( $p$ for trend $<0.001$ for all) (Appendix 1, Supplemental Figure 4). For triglycerides and remnant cholesterol, U-shaped curves were observed with the highest levels in $\varepsilon 22$ and $\varepsilon 44$ carriers ( $p<0.001$ for both). Appendix 1, Supplemental Figure 5, shows all 9 possible genotype combinations. Owing to rarity, $\varepsilon 442, \varepsilon 422$ and $\varepsilon 4422$ were excluded from further analyses.

Other covariates independently associated with an increased risk of all cerebrovascular disease included lipid-lowering therapy (adjusted HR $2.25,95 \% \mathrm{Cl} 2.13-2.38$ ), atrial fibrillation (adjusted HR 1.52, 95\% Cl 1.44-1.61), smoking (adjusted HR
1.49, 95\% Cl 1.41-1.57), hypertension (adjusted HR 1.17, 95\% Cl $1.11-1.24$ ) and physical inactivity (adjusted HR $1.13,95 \% \mathrm{Cl}$ $1.08-1.18$ ). In contrast, male sex (adjusted HR $0.64,95 \% \mathrm{Cl} 0.57$ 0.73 ) was associated with a decreased risk of all cerebrovascular disease (Table 2).

\section{Interpretation}

We found that the absolute 10-year risk of dementia for 844 carriers is modified by age and sex. Absolute 10-year risk increased from $6 \%-7 \%$ at age $60-69$ years up to $19 \%-24 \%$ at age 80 years and older for Alzheimer disease, and from $8 \%-10 \%$ up to $33 \%-$ $38 \%$ for all dementia, respectively. Such estimates have the potential to facilitate the identification of high-risk individuals for targeted preventive interventions.

Our study provides age- and sex-specific absolute 10-year risk as a function of each of the 6 common APOE genotypes based on a large general population sample with more than 3000 ع44 carriers. Absolute risk was recently estimated in 4 different studies with a total of 392 \&44 carriers, but with no sex stratification 


\section{Alzheimer disease}

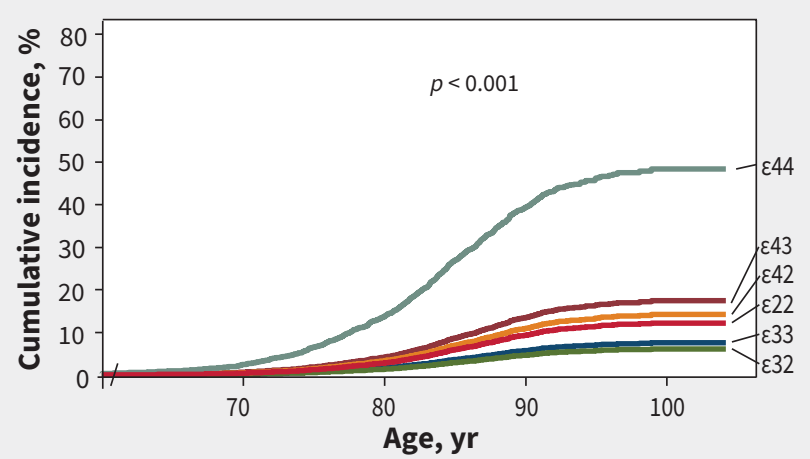

Unspecified dementia

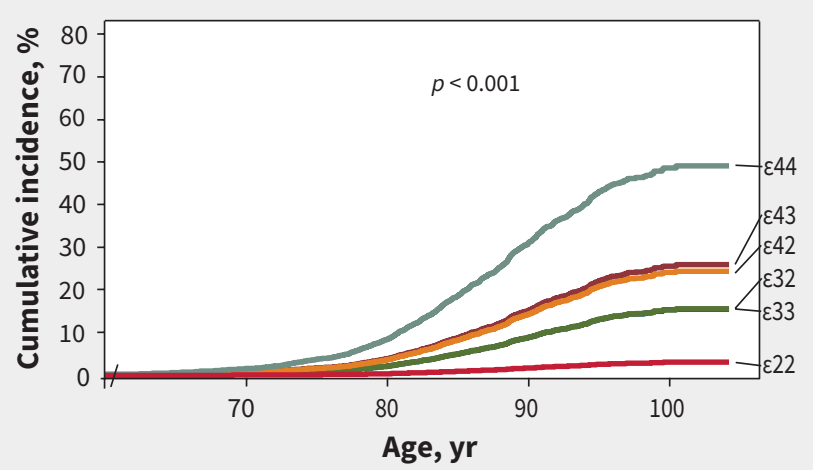

\section{Vascular dementia}

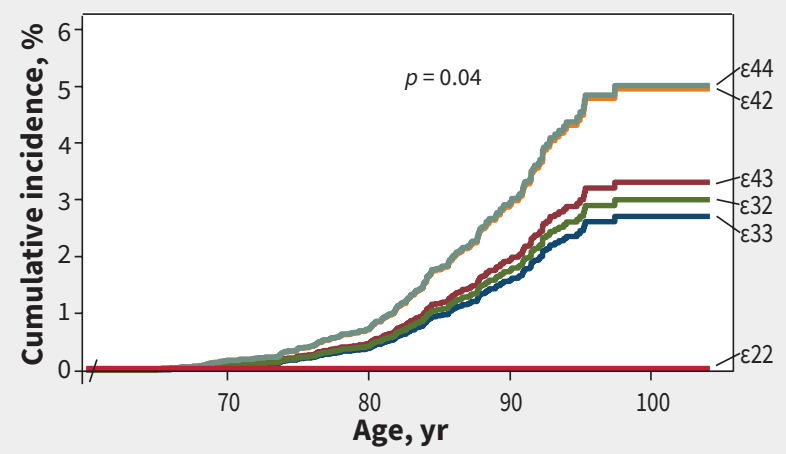

All dementia

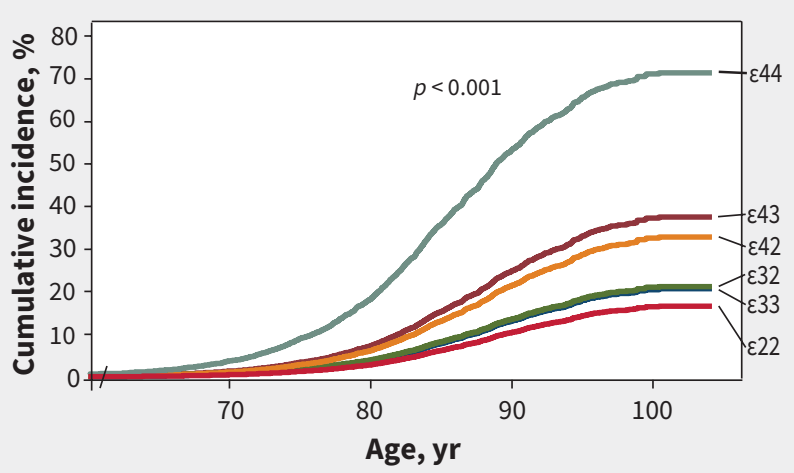

Figure 2: Cumulative incidence of dementia, by the method of Fine and Gray, ${ }^{24}$ as a function of age and APOE genotype. The $p$ values are trends across genotypes from $\varepsilon 22$ to $\varepsilon 32$ to $\varepsilon 33$ to $\varepsilon 42$ to $\varepsilon 43$ to $\varepsilon 44$ for the unadjusted competing risk regression (age as time scale).

owing to a lack of power. ${ }^{25}$ Sex-specific estimates for absolute risk are important because of differences in life span between the sexes and because the effect of $A P O E$ genotype may be modified by sex, ${ }^{25}$ where age-dependent sex hormone changes in apoEmediated lipid metabolism could have an influence. ${ }^{26}$ Because dementia at present remains effectively untreatable, reducing the risk is of major importance. A third of all cases of dementia has recently been calculated to be preventable with early intervention for hypertension, smoking, diabetes, obesity, depression and hearing loss. ${ }^{3}$ Further, declines in incidences in some highincome countries have been suggested to be caused by improved prevention and treatment of vascular risk factors. ${ }^{27,28}$ Therefore, robust absolute risk estimates will be pivotal instruments for identification of high-risk individuals who will benefit the most from targeted preventive intervention.

The present data also illustrate how the $\varepsilon 4$ allele of the $A P O E$ polymorphism affects risk of both dementia and cerebrovascular disease in a dose-dependent manner, consistent with the existing literature. ${ }^{5,9,16-19,22,25}$ The data on subtypes of dementia and cerebrovascular disease show that the $\varepsilon 42$ genotype is more important than the 843 genotype for vascular components of dementia, as well as for cerebrovascular disease, than previously reported. ${ }^{11-19}$ This is biologically plausible, given that the $\varepsilon 42$ genotype is a combination of 2 hyperlipidemic alleles: $\varepsilon 2$ has low receptor affinity for the LDL receptor, resulting in susceptibility to type III hyperlipoproteinemia with increased levels of triglycerides and remnant particles, ${ }^{7,29}$ whereas $\varepsilon 4$ is associated with increased plasma levels of LDL cholesterol and triglycerides. ${ }^{30}$ The decreased receptor affinity for apoE2 leads to a decreased apoE-mediated uptake and an upregulation of the LDL receptor, probably explaining the associated low LDL cholesterol and high triglyceride levels. ${ }^{31}$ The preference of apoE4 for very low-density lipoprotein in plasma increases hepatic clearance and results in lower apoE levels, subsequently causing downregulation of LDL receptors and corresponding increases in plasma levels of LDL cholesterol. ${ }^{7,32}$ The biological mechanism behind increased triglyceride levels in $\varepsilon 4$ carriers is not well understood. Further, given that stroke and cerebrovascular disease likely make the brain more prone to dementia, 33,34 we assessed the genotype-specific risk pattern for all dementia after a diagnosis of cerebrovascular disease. We found that $\varepsilon 44$ and $\varepsilon 42$ were robustly associated with the highest risk, consistent with the vascular effects of $\varepsilon 42$, and in accordance with earlier reports in smaller studies. ${ }^{33,34}$

The underlying mechanisms for our findings are only partly understood. Likely biological explanations involve direct 


\begin{tabular}{lccc}
$\begin{array}{l}\text { APOE } \\
\text { genotype }\end{array}$ & $\begin{array}{c}\boldsymbol{n} \\
\text { total }\end{array}$ & $\begin{array}{c}\boldsymbol{n} \\
\text { events }\end{array}$ & $\begin{array}{c}\text { HR } \\
(\mathbf{9 5 \%} \mathrm{CI})\end{array}$ \\
\hline \multicolumn{2}{l}{ Alzheimer disease } & & \\
$\varepsilon 22$ & 715 & 6 & $1.30(0.58-2.91)$ \\
$\varepsilon 32$ & 12994 & 59 & $0.62(0.47-0.82)$ \\
$\varepsilon 33$ & 58172 & 405 & 1.00 (Ref.) \\
$\varepsilon 42$ & 3013 & 28 & $1.48(1.01-2.16)$ \\
$\varepsilon 43$ & 26626 & 398 & $2.47(2.15-2.84)$ \\
$\varepsilon 44$ & 3017 & 112 & $8.74(7.08-10.79)$
\end{tabular}

Vascular dementia

$\begin{array}{cccc}\varepsilon 22 & 715 & 0 & \text { (No events) } \\ \varepsilon 32 & 12994 & 30 & 1.06(0.71-1.58) \\ \varepsilon 33 & 58172 & 119 & 1.00 \text { (Ref.) } \\ \varepsilon 43 & 26626 & 77 & 1.66(1.24-2.21) \\ \varepsilon 42 & 3013 & 11 & 1.99(1.07-3.70) \\ \varepsilon 44 & 3017 & 11 & 2.87(1.54-5.33)\end{array}$

\section{Unspecified dementia}

$\begin{array}{cccc}\varepsilon 22 & 715 & 1 & 0.13(0.02-0.92) \\ \varepsilon 32 & 12994 & 126 & 0.81(0.67-0.98) \\ \varepsilon 33 & 58172 & 641 & 1.00 \text { (Ref.) } \\ \varepsilon 42 & 3013 & 40 & 1.33(0.97-1.83) \\ \varepsilon 43 & 26626 & 457 & 1.85(1.64-2.09) \\ \varepsilon 44 & 3017 & 89 & 4.68(3.74-5.85)\end{array}$

\section{All dementia}

$\begin{array}{lccc}\varepsilon 22 & 715 & 7 & 0.60(0.29-1.27) \\ \varepsilon 32 & 12994 & 187 & 0.80(0.69-0.94) \\ \varepsilon 33 & 58172 & 974 & 1.00 \text { (Ref.) } \\ \varepsilon 42 & 3013 & 62 & 1.34(1.04-1.74) \\ \varepsilon 43 & 26626 & 764 & 2.03(1.85-2.23) \\ \varepsilon 44 & 3017 & 166 & 5.77(4.89-6.81)\end{array}$

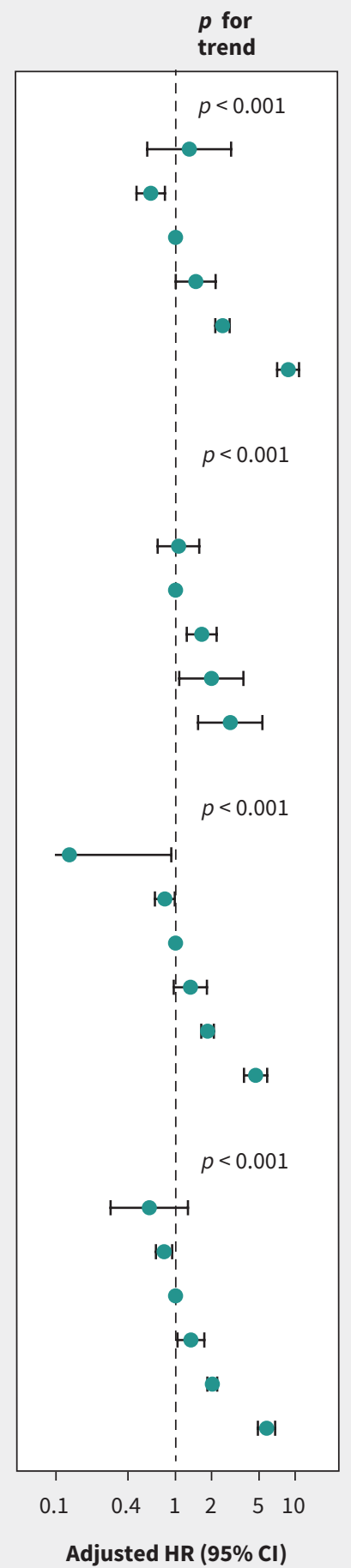

Figure 3: Risk of dementia as a function of $A P O E$ genotype. Hazard ratios (HRs) were adjusted for age (time scale), sex, body mass index, hypertension, diabetes mellitus, smoking, alcohol consumption, physical inactivity, menopausal status and hormonal replacement therapy (only women), lipidlowering therapy and education. The $p$ values are trends across genotypes from $\varepsilon 22$ to $\varepsilon 32$ to $\varepsilon 33$ to $\varepsilon 42$ to $\varepsilon 43$ to $\varepsilon 44$, except for vascular dementia, where trend was calculated from $\varepsilon 22$ to $\varepsilon 32$ to $\varepsilon 33$ to $\varepsilon 43$ to $\varepsilon 42$ to $\varepsilon 44 . \mathrm{Cl}=$ confidence interval, Ref. = reference. 
Table 2: Hazard ratios for covariates in multivariable-adjusted Cox regression models for all dementia and all cerebrovascular disease

HR $(95 \% \mathrm{CI})$

\section{Covariate}

APOE genotype

Male sex

Body mass index

Lowest tertile (CCHS: <23.3; CGPS: <24.0)

Middle tertile (CCHS: 23.3-26.8; CGPS: 24.0-27.4)

Highest tertile (CCHS: > 26.8; CGPS: > 27.4)

Hypertension*

Diabetes mellitus $\dagger$

Smoking

Alcohol consumption§

Physical inactivity 9

Postmenopausal status and hormonal replacement therapy*

Premenopausal

Postmenopausal without hormonal replacement therapy

Postmenopausal with hormonal replacement therapy

Lipid-lowering therapy ${ }^{\dagger} \dagger$

Education $<8 \mathrm{yr}$

Atrial fibrillation $\ddagger \ddagger$
All dementia

$\varepsilon 22: 0.60(0.29-1.27)$

ع32: 0.80 (0.69-0.94)

ع33: 1.00 (Ref.)

ع42: $1.34(1.04-1.74)$

ع43: 2.03 (1.85-2.23)

ع44: 5.77 (4.89-6.81)

$0.62(0.40-0.96)$

1.00 (Ref.)

$0.97(0.88-1.08)$

$0.96(0.86-1.07)$

$0.82(0.74-0.91)$

$1.30(1.09-1.55)$

$1.68(1.52-1.85)$

$1.00(0.89-1.12)$

$1.16(1.06-1.27)$

$$
\begin{gathered}
1.00 \text { (Ref.) } \\
0.65(0.42-1.00) \\
0.72(0.46-1.13) \\
0.81(0.71-0.93) \\
1.34(1.23-1.47)
\end{gathered}
$$

\section{All cerebrovascular disease}

ع22: 0.89 (0.66-1.21)

ع32: 1.00 (0.93-1.08)

ع33: 1.00 (Ref.)

ع43: 1.01 (0.95-1.06)

ع42: $1.17(1.03-1.34)$

ع44: 1.30 (1.15-1.48)

$0.64(0.57-0.73)$

1.00 (Ref.)

$0.98(0.93-1.04)$

$1.04(0.98-1.10)$

1.17 (1.11-1.24)

$1.02(0.93-1.11)$

1.49 (1.41-1.57)

$0.97(0.92-1.03)$

$1.13(1.08-1.18)$

$$
1.00 \text { (Ref.) }
$$

$0.47(0.42-0.54)$

$0.53(0.46-0.62)$

$2.25(2.13-2.38)$

$1.02(0.97-1.07)$

$1.52(1.44-1.61)$

Note: $\mathrm{CCHS}=$ Copenhagen City Heart Study, CGPS = Copenhagen General Population Study, $\mathrm{Cl}=$ confidence interval, Ref. $=$ reference.

*Hypertension was defined by use of antihypertensive medication, systolic blood pressure of $\geq 140 \mathrm{~mm} \mathrm{Hg}$, and/or diastolic blood pressure of $\geq 90 \mathrm{~mm} \mathrm{Hg}$.

†Diabetes mellitus was defined by self-reported disease, use of insulin or oral hypoglycemic agents, and/or nonfasting plasma glucose level of $>11 \mathrm{mmol} / \mathrm{L}$ (> $198 \mathrm{mg} / \mathrm{dL}$ ).

¥Smoking was defined by current smoking.

$\S$ Alcohol consumption was defined by $>14 / 21$ units per week for women/men ( 1 unit $=12 \mathrm{~g}$ alcohol, equivalent to $1 \mathrm{glass}$ of wine or spirit or 1 beer [ $33 \mathrm{cL}=330 \mathrm{~mL}$ ).

IPhysical inactivity was defined by $\leq 4$ hours per week of light physical activity in leisure time.

**Women reported menopausal status and use of hormonal replacement therapy. The individual hazard ratios for postmenopausal status and hormonal replacement therapy should be interpreted with caution, because the covariate in itself reflects age, and these estimates are from the full model with adjustment for age as the time scale. Adjusting for postmenopausal status and hormonal replacement therapy as a covariate is still appropriate, and the inclusion of this does not change the genetic estimates (Appendix 1 , Supplemental Table 1).

††Lipid-lowering therapy primarily consisted of statins (yes/no).

$\ddagger \ddagger$ Atrial fibrillation was defined by any diagnosis of atrial fibrillation ever.

effects in brain tissue, and blood-brain barrier and vascular effects subsequently affecting brain functions. Brain apoE mediates clearance of amyloid- $\beta$, which exerts its toxic function by accumulating in brain tissue. Clearance of amyloid- $\beta$ is decreased in Alzheimer disease, ${ }^{6,35}$ and the $\varepsilon 4$ allele is associated with increased brain amyloid burden in a dose-dependent manner in healthy older individuals and in patients with mild cognitive impairment. ${ }^{36,37}$ These human findings are supported by apoE isoform-specific effects on both amyloid- $\beta$ oligomer formation and plaque deposition in animal models. ${ }^{38-40}$ Accumulation of amyloid- $\beta$ in the cerebral vasculature causes cerebral amyloid angiopathy, ${ }^{5}$ and both $\varepsilon 4$ and $\varepsilon 2$ are associated with increased risk of lobar intracerebral hemorrhage related to cerebral amyloid angiopathy. ${ }^{41-43}$ Among the subtypes of cerebrovascular disease in the present study, the highest risk for $\varepsilon 44$ carriers is observed for hemorrhagic stroke, supporting the hypothesis that the $\varepsilon 4$ allele increases cerebral vasculature fragility. It is likely that deposition of amyloid- $\beta$ in arterioles in the central nervous system combined with degradation of the blood-brain barrier altogether leaves a rather frail intracerebral vasculature prone to cerebrovascular disease. ${ }^{5,44,45}$ This concept is supported by previous studies and the present largescale general population study on major subtypes of cerebrovascular disease. Finally, an atherogenic lipid profile is a wellknown risk factor for cerebrovascular disease, ${ }^{46}$ and both $\varepsilon 22$ homozygosity and the $\varepsilon 4$ allele are associated with hyperlipidemia. This is in line with the present observations that the $\varepsilon 42$ genotype confers higher risk in cerebrovascular disease and vascular dementia (i.e., diseases with vascular components) than previously assumed. 


\section{Limitations}

The generalizability of our study is limited by the inclusion of only white individuals. $A P O E$ allele frequencies vary between ancestries, and $A P O E$ genotypes may not be associated with adverse outcomes in all populations. ${ }^{7}$ Another potential limitation is information on dementia diagnoses, where misclassification in the outcome may be present. This might explain the similar directions of the effects of $A P O E$ genotype on Alzheimer disease and all dementia. A previous thorough examination of 200 dementia diagnoses from the national registries showed after a full clinical workup that $33 \%$ of cases of Alzheimer disease were misclassified as dementia without specification (which is a subgroup of our all-dementia diagnosis). However, once the Alzheimer disease diagnosis was entered in the registries, it was correct in $81 \%$ of the cases. ${ }^{47}$ Consequently, the misclassified fraction of Alzheimer disease is most likely included in our all-dementia diagnosis, and will only bias our results for Alzheimer disease toward the null hypothesis, making these estimates conservative. These issues with potential misclassification emphasize the rationale for presenting results on both Alzheimer disease and all dementia, as we have done. Further, we ensured that about $80 \%$ of observations from 1996 were obtained at specialized units by scoring all individual Alzheimer-disease and all-dementia events in our cohorts. ${ }^{23}$

\section{Conclusion}

We found that the absolute 10-year risk of dementia for $\varepsilon 44$ carriers is modified by age and sex. Absolute 10 -year risk increased from $6 \%-7 \%$ at age $60-69$ years up to $19 \%-24 \%$ at age 80 years and older for Alzheimer disease, and from $8 \%-10 \%$ up to $33 \%-$ $38 \%$ for all dementia, respectively. The present absolute 10-year risk estimates of dementia by age, sex and APOE genotype have the potential to identify high-risk individuals for early targeted preventive interventions. Taken together, the present findings emphasize $A P O E$ genotype as an important component in the individual risk assessment of dementia and cerebrovascular disease.

\section{References}

1. Mortality and Cause of Death Collaborators. Global, regional, and national agesex specific all-cause and cause-specific mortality for 240 causes of death, 1990-2013: a systematic analysis for the Global Burden of Disease Study 2013. Lancet 2015;385:117-71.

2. Prince $M$, Bryce R, Albanese $E$, et al. The global prevalence of dementia: a systematic review and metaanalysis. Alzheimers Dement 2013;9:63-75e2.

3. Livingston $\mathrm{G}$, Sommerlad A, Orgeta V, et al. Dementia prevention, intervention, and care. Lancet 2017;390:2673-734.

4. Vos T, Flaxman AD, Naghavi M, et al. Years lived with disability (YLDs) for 1160 sequelae of 289 diseases and injuries 1990-2010: a systematic analysis for the Global Burden of Disease Study 2010. [published erratum in Lancet 2013; 381:628]. Lancet 2012;380:2163-96.

5. Verghese PB, Castellano JM, Holtzman DM. Apolipoprotein E in Alzheimer's disease and other neurological disorders. Lancet Neurol 2011;10:241-52.

6. Cramer PE, Cirrito JR, Wesson DW, et al. ApoE-directed therapeutics rapidly clear beta-amyloid and reverse deficits in AD mouse models. Science 2012; 335:1503-6.

7. Mahley RW, Rall SC Jr. Type III hyperlipoproteinemia (dysbetalipoproteinemia): the role of apolipoprotein $\mathrm{E}$ in normal and abnormal lipoprotein metabolism. In: Scriver CR, Beaudet AL, Sly WS, et al., editors. The metabolic and molecular bases of inherited disease. 8th ed. New York: McGraw-Hill; 2001:2835-62.
8. Hort J, O'Brien JT, Gainotti G, et al. EFNS guidelines for the diagnosis and management of Alzheimer's disease. Eur J Neurol 2010;17:1236-48.

9. Corder EH, Saunders AM, Strittmatter WJ, et al. Gene dose of apolipoprotein E type 4 allele and the risk of Alzheimer's disease in late onset families. Science 1993;261:921-3.

10. Lambert JC, Ibrahim-Verbaas CA, Harold D, et al. Meta-analysis of 74046 individuals identifies 11 new susceptibility loci for Alzheimer's disease. Nat Genet 2013;45:1452-8.

11. Yin YW, Li JC, Wang JZ, et al. Association between apolipoprotein E gene polymorphism and the risk of vascular dementia: a meta-analysis. Neurosci Lett 2012;514:6-11.

12. Liu X, Li L, Liu F, et al. ApoE gene polymorphism and vascular dementia in Chinese population: a meta-analysis. J Neural Transm (Vienna) 2012;119:387-94.

13. Chuang YF, Hayden KM, Norton MC, et al. Association between APOE epsilon4 allele and vascular dementia: the Cache County study. Dement Geriatr Cogn Disord 2010;29:248-53.

14. Baum L, Lam LC, Kwok T, et al. Apolipoprotein E epsilon4 allele is associated with vascular dementia. Dement Geriatr Cogn Disord 2006;22:301-5.

15. Mou C, Han T, Wang M, et al. Correlation of polymorphism of APOE and LRP genes to cognitive impairment and behavioral and psychological symptoms of dementia in Alzheimer's disease and vascular dementia. Int J Clin Exp Med 2015;8:21679-83.

16. Chen C, Hu Z, Apo E. Polymorphisms and the risk of different subtypes of stroke in the Chinese population: a comprehensive meta-analysis. Cerebrovasc Dis 2016;41:119-38.

17. Zhang R, Wang X, Tang Z, et al. Apolipoprotein E gene polymorphism and the risk of intracerebral hemorrhage: a meta-analysis of epidemiologic studies. Lipids Health Dis 2014;13:47.

18. Sudlow C, Martinez Gonzalez NA, Kim J, et al. Does apolipoprotein E genotype influence the risk of ischemic stroke, intracerebral hemorrhage, or subarachnoid hemorrhage? Systematic review and meta-analyses of 31 studies among 5961 cases and 17,965 controls. Stroke 2006;37:364-70.

19. Khan TA, Shah T, Prieto D, et al. Apolipoprotein E genotype, cardiovascular biomarkers and risk of stroke: systematic review and meta-analysis of 14015 stroke cases and pooled analysis of primary biomarker data from up to 60883 individuals. Int J Epidemiol 2013;42:475-92.

20. Frikke-Schmidt R, Nordestgaard BG, Stene MC, et al. Association of loss-offunction mutations in the ABCA1 gene with high-density lipoprotein cholesterol levels and risk of ischemic heart disease. JAMA 2008;299:2524-32.

21. Jørgensen AB, Frikke-Schmidt R, Nordestgaard BG, et al. Loss-of-function mutations in APOC3 and risk of ischemic vascular disease. N Engl J Med 2014; 371:32-41.

22. Rasmussen KL, Tybjaerg-Hansen A, Nordestgaard BG, et al. Plasma levels of apolipoprotein E and risk of dementia in the general population. Ann Neurol 2015;77:301-11.

23. Rasmussen KL, Tybjaerg-Hansen A, Nordestgaard BG, et al. Plasma apolipoprotein $\mathrm{E}$ levels and risk of dementia: a Mendelian randomization study of 106562 individuals. Alzheimers Dement 2018;14:71-80.

24. Fine JP, Gray RJ. A proportional hazards model for the subdistribution of a competing risk. J Am Stat Assoc 1999;94:496-509.

25. Qian J, Wolters FJ, Beiser A, et al. APOE-related risk of mild cognitive impair ment and dementia for prevention trials: an analysis of four cohorts. PLoS Med 2017;14:e1002254.

26. Phillips NR, Havel RJ, Kane JP. Sex-related differences in the concentrations of apolipoprotein $\mathrm{E}$ in human blood plasma and plasma lipoproteins. J Lipid Res 1983;24:1525-31.

27. Satizabal CL, Beiser AS, Chouraki V, et al. Incidence of dementia over three decades in the Framingham Heart Study. N Engl J Med 2016;374:523-32.

28. Ahmadi-Abhari S, Guzman-Castillo M, Bandosz P, et al. Temporal trend in dementia incidence since 2002 and projections for prevalence in England and Wales to 2040: modelling study. BMJ 2017;358:j2856.

29. Utermann G, Hees M, Steinmetz A. Polymorphism of apolipoprotein E and occurrence of dysbetalipoproteinaemia in man. Nature 1977;269:604-7.

30. Bennet AM, Di AE, Ye Z, et al. Association of apolipoprotein E genotypes with lipid levels and coronary risk. JAMA 2007;298:1300-11.

31. Boerwinkle E, Utermann G. Simultaneous effects of the apolipoprotein E polymorphism on apolipoprotein $\mathrm{E}$, apolipoprotein $\mathrm{B}$, and cholesterol metabolism. Am J Hum Genet 1988;42:104-12. 
32. Mahley RW. Central nervous system lipoproteins: ApoE and regulation of cholesterol metabolism. Arterioscler Thromb Vasc Biol 2016;36:1305-15.

33. Fekih-Mrissa N, Klai S, Mrad M, et al. Apolipoprotein E genotypes associated with Alzheimer disease and concomitant stroke. J Stroke Cerebrovasc Dis 2014;23:681-5.

34. Tosto G, Bird TD, Bennett DA, et al. The role of cardiovascular risk factors and stroke in familial Alzheimer disease. JAMA Neurol 2016;73:1231-7.

35. Mawuenyega KG, Sigurdson W, Ovod V, et al. Decreased clearance of CNS betaamyloid in Alzheimer's disease. Science 2010;330:1774.

36. Villemagne VL, Pike KE, Chetelat G, et al. Longitudinal assessment of Abeta and cognition in aging and Alzheimer disease. Ann Neurol 2011;69:181-92.

37. Reiman EM, Chen K, Liu X, et al. Fibrillar amyloid-beta burden in cognitively normal people at 3 levels of genetic risk for Alzheimer's disease. Proc Natl Acad Sci U S A 2009;106:6820-5.

38. Castellano JM, Kim J, Stewart FR, et al. Human apoE isoforms differentially regulate brain amyloid-beta peptide clearance. Sci Transl Med 2011;3:89ra57.

39. Yu JT, Tan L, Hardy J. Apolipoprotein E in Alzheimer's disease: an update. Annu Rev Neurosci 2014;37:79-100.
40. Bales KR, Liu F, Wu S, et al. Human APOE isoform-dependent effects on brain beta-amyloid levels in PDAPP transgenic mice. J Neurosci 2009;29:6771-9.

41. Greenberg SM, Rebeck GW, Vonsattel JP, et al. Apolipoprotein E epsilon 4 and cerebral hemorrhage associated with amyloid angiopathy. Ann Neurol 1995;38:254-9.

42. Nicoll JA, Burnett C, Love S, et al. High frequency of apolipoprotein E epsilon 2 allele in hemorrhage due to cerebral amyloid angiopathy. Ann Neurol 1997; 41:716-21.

43. Biffi A, Sonni A, Anderson CD, et al. Variants at APOE influence risk of deep and lobar intracerebral hemorrhage. Ann Neurol 2010;68:934-43.

44. Zlokovic BV. Neurovascular pathways to neurodegeneration in Alzheimer's disease and other disorders. Nat Rev Neurosci 2011;12:723-38.

45. Bell RD, Winkler EA, Singh I, et al. Apolipoprotein E controls cerebrovascular integrity via cyclophilin A. Nature 2012;485:512-6.

46. European Stroke Organisation (ESO) Executive Committee; ESO Writing Committee. Guidelines for management of ischaemic stroke and transient ischaemic attack 2008. Cerebrovasc Dis 2008;25:457-507.

47. Phung TK, Andersen BB, Hogh P, et al. Validity of dementia diagnoses in the Danish hospital registers. Dement Geriatr Cogn Disord 2007;24:220-8.

\section{Competing interests: None declared.}

This article has been peer reviewed.

Affiliations: Department of Clinical Biochemistry (Rasmussen, Tybjærg-Hansen, Frikke-Schmidt), Rigshospitalet, Copenhagen University Hospital, Copenhagen, Denmark; Department of Clinical Biochemistry (Rasmussen, Nordestgaard), Herlev and Gentofte Hospital, Copenhagen University Hospital, Herlev, Denmark.

Contributors: Katrine Rasmussen and Ruth Frikke-Schmidt contributed to the study concept and design, acquisition of data, statistical analysis and interpretation of data. Anne Tybjærg-Hansen contributed to the study concept and design, and acquisition of data. Børge G. Nordestgaard contributed to acquisition of data. Anne Tybjærg-Hansen, Børge G.
Nordestgaard and Ruth Frikke-Schmidt obtained funding and gave administrative, technical and material support. Ruth FrikkeSchmidt supervised the study. Katrine Rasmussen and Ruth Frikke-Schmidt drafted the manuscript, which all of the authors revised. All of the authors gave final approval of the version to be published and agreed to be accountable for all aspects of the work.

Funding: This work was supported by the Danish Council for Independent Research (grant no. 10-081618), the Research Council at Rigshospitalet, the Lundbeck Foundation, the Danish Alzheimer Research Fund, M.L. Jørgensen \& Gunnar Hansen's Fund, and the Research Fund at the Capital Region of Denmark. The funding sources had no role in the design and conduct of the study; collection, management, analysis and interpretation of the data; preparation, review and approval of the manuscript; or decision to submit the manuscript for publication.

Acknowledgments: The authors thank staff and participants of the Copenhagen General Population Study and the Copenhagen City Heart Study for their important contributions.

Data sharing: Upon reasonable request to the corresponding author, data are available for collaborative projects.

Accepted: June 27, 2018

Correspondence to: Ruth Frikke-Schmidt, ruth.frikke-schmidt@regionh.dk 